\title{
Optimization of Microphone Locations for Acoustic Liner Impedance Eduction
}

\author{
M. G. Jones*and W. R. Watson ${ }^{\dagger}$ \\ NASA Langley Research Center, Hampton, VA 23681 \\ and \\ J. C. June \\ University of Florida, Gainesville, FL 32605
}

\begin{abstract}
Two impedance eduction methods are explored for use with data acquired in the NASA Langley Grazing Flow Impedance Tube. The first is an indirect method based on the convected Helmholtz equation, and the second is a direct method based on the Kumaresan and Tufts algorithm. Synthesized no-flow data, with random jitter to represent measurement error, are used to evaluate a number of possible microphone locations. Statistical approaches are used to evaluate the suitability of each set of microphone locations. Given the computational resources required, small sample statistics are employed for the indirect method. Since the direct method is much less computationally intensive, a Monte Carlo approach is employed to gather its statistics. A comparison of results achieved with full and reduced sets of microphone locations is used to determine which sets of microphone locations are acceptable. For the indirect method, each array that includes microphones in all three regions (upstream and downstream hard wall sections, and liner test section) provides acceptable results, even when as few as eight microphones are employed. The best arrays employ microphones well away from the leading and trailing edges of the liner. The direct method is constrained to use microphones opposite the liner. Although a number of arrays are acceptable, the optimum set employs 14 microphones positioned well away from the leading and trailing edges of the liner. The selected sets of microphone locations are also evaluated with data measured for ceramic tubular and perforate-over-honeycomb liners at three flow conditions (Mach 0.0, 0.3, and 0.5). They compare favorably with results attained using all 53 microphone locations. Although different optimum microphone locations are selected for the two impedance eduction methods, there is significant overlap. Thus, the union of these two microphone arrays is preferred, as it supports usage of both methods. This array contains 3 microphones in the upstream hard wall section, 14 microphones opposite the liner, and 3 microphones in the downstream hard wall section.
\end{abstract}

\section{Introduction}

Aircraft noise can conveniently be divided into two components, propulsion noise and airframe noise. Of these, propulsion noise is dominant for the takeoff condition, and is similar in magnitude with airframe noise for the approach condition. ${ }^{1}$ One of the key components of propulsion noise is fan noise, which has typically been controlled via modifications to the fan (e.g., adjustments to the lean and sweep of the fan blades) and the installation of acoustic liners in the walls of the engine nacelle. These acoustic liners are the focus of the current study. The key parameter used to evaluate these liners is the acoustic surface impedance, computed as the ratio of the acoustic pressure to the normal component of acoustic particle velocity at the surface of the liner. The focus of the current research is the optimization of tools used to determine the acoustic impedance of these acoustic liners.

*Senior Research Scientist, Research Directorate, Structural Acoustics Branch, AIAA Associate Fellow.

${ }^{\dagger}$ Senior Research Scientist, Research Directorate, Computational AeroSciences Branch, AIAA Associate Fellow.

${ }^{\ddagger} \mathrm{PhD}$ candidate. 
Extensive research over the last four decades has been devoted to evaluation of the acoustic impedance of liners in the presence of grazing flow. ${ }^{2-16}$ This research area has been a particular focus for the NASA Langley Research Center (LaRC) liner physics team for much of this time. The LaRC team has implemented a number of methods, both indirect ${ }^{14}$ and direct ${ }^{8}$ for acoustic impedance eduction. One of these methods ${ }^{14}$ has served as the foundation for much of this research. This method is based on the convected Helmholtz equation (CHE), assumes the flow over the liner surface to be uniform, and educes a uniform impedance (in its current implementation) at the liner surface. Two of the salient features of this method are its ability to account for (1) multiple modes over the extent of the acoustic liner, and (2) interactive effects between the acoustic liner and the hard wall sections upstream and downstream of the liner.

The impedance eduction methods studied by the LaRC team are based on measurements acquired in the Grazing Flow Impedance Tube (GFIT). The GFIT allows for convenient evaluation of acoustic liners mounted in the test window upper wall with lengths of $51 \mathrm{~mm}$ to $610 \mathrm{~mm}$, at grazing flow Mach numbers up to 0.6 , and with source sound pressure levels (SPL) of up to $155 \mathrm{~dB}$ over a frequency range of 0.4 to $3.0 \mathrm{kHz}$. The GFIT test window contains 53 microphones flush-mounted in the lower wall for use with the CHE impedance eduction method.

Data published by LaRC has also been used by others as a basis for evaluation of their impedance eduction methods. ${ }^{15,17,18}$ Due to multiple constraints (e.g., cost and space limitations), many of these studies have sought to demonstrate the ability to achieve satisfactory results with a limited number of microphones. These comparison studies have provided valuable insights to the liner community, and have demonstrated that much information can be gleaned with a limited number of microphones.

The goal of the current study is to determine the minimum number of microphones for use with two impedance eduction methods, one direct [the Kumaresan and Tufts (KT) algorithm] and the other indirect [the convected Helmholtz equation (CHE) method]. The KT algorithm extracts the axial propagation constant from sound pressure level and phase information measured over the axial extent of the liner, and then uses this axial propagation constant with a normal mode solution to the convected Helmholtz equation and the local-reacting wall impedance boundary condition to compute the acoustic impedance of the liner. ${ }^{19}$ The CHE method educes the impedance by mimimizing an objective function that is the difference between the measured SPL and phase profiles and those predicted by a finite element solution to the convected Helmholtz equation. ${ }^{14}$

Synthesized data (SPL and phase profiles) for two liner configurations are used in this study. These data are adjusted to include random jitter consistent with measurement error typically experienced for measurements in the GFIT, and the effects of this random error on the educed impedance spectra are evaluated. This process is performed multiple times to generate a number of data sets for evaluation. For the KT algorithm, a Monte-Carlo process based on a large number of data sets $(20,000)$ is used to evaluate the uncertainty in the results. For the CHE method, which requires significantly more computational resources than the KT algorithm, eleven randomized data sets and $95 \%$ confidence intervals are used to evaluate the uncertainty in the results. These evaluations are first conducted with the full set of microphone locations currently used in the GFIT ( 53 for the CHE method, over the length of the test window; 25 for the KT algorithm, over the length of the lined portion of the test window). These impedance eductions are repeated with data removed for selected microphone locations, and the results are compared against those results achieved with the full set of microphone locations. This process is continued (additional microphone locations removed) to determine the optimum microphone locations (smallest number, best locations) for each of the impedance eduction methods.

Section II provides a description of the test liners used in this study and a brief description of the Grazing Flow Impedance Tube (GFIT), which is used as the basis for the optimization study. Section III provides a description of the two impedance eduction methods considered in this study. Key results are provided in Section IV, and concluding remarks are presented in Section V.

\section{Experimental Method}

Simulated and measured acoustic pressure datasets for the NASA Langley Research Center grazing flow impedance tube (GFIT) are used throughout this study. Simulated data based on previous GFIT tests with two wire mesh-over-honeycomb liners are used to determine which of the GFIT microphones are required to achieve acceptable impedance eductions in a no-flow environment. Data acquired at the selected microphone 
locations with ceramic tubular and conventional liners mounted in the GFIT are then used to confirm the validity of the selected microphone locations. This validation is conducted both without and with mean flow.

\section{A. Grazing Flow Impedance Tube (GFIT)}

The GFIT (see Fig. 1) has a cross-sectional geometry of $50.8 \mathrm{~mm}$ wide by $63.5 \mathrm{~mm}$ high, such that higherorder modes in the horizontal and vertical dimensions cut on at different frequencies. It allows evaluation of acoustic liners with lengths from $50.8 \mathrm{~mm}$ to $609.6 \mathrm{~mm}$. The surface of the test liner forms a portion of the upper wall of the test window. Sound is generated upstream of the source plane, and air flows left to right through the test section. Fifty-three microphones flush-mounted in the lower wall (opposite the liner) are used to measure the acoustic pressure field over the axial extent of $0 \leq x \leq L$ (see Fig. 1).

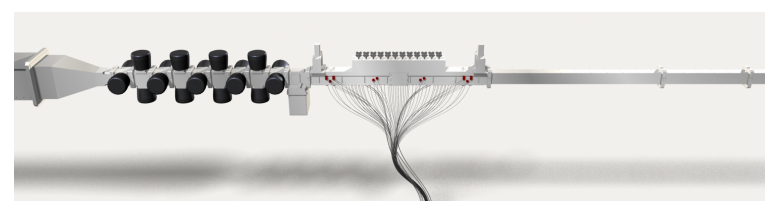

(a) Sketch of GFIT

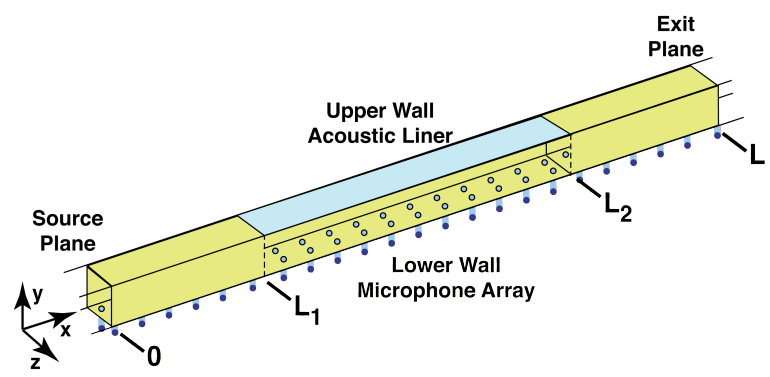

(b) Sketch of GFIT test section.

Figure 1: NASA LaRC Grazing Flow Impedance Tube (GFIT).

\section{B. Test Liners - Simulated Data}

Two liners are considered for the initial portion of this study (see Fig. 2). Each contains a wire mesh facesheet with a DC flow resistance of 270 MKS Rayls mounted onto a cavity filled with a honeycomb core and sealed with a rigid back plate. The first liner (TL1) has a cavity depth of $38.1 \mathrm{~mm}$, and the second (TL2) has a cavity depth of $76.2 \mathrm{~mm}$. These liner configurations were chosen because they can be easily modeled ${ }^{16}$ as

$$
\zeta(f)=R_{f}-i \cot (k d)
$$

where $R_{f}$ is the DC flow resistance (270 MKS Rayls for these liners), $f$ is the frequency, $k$ is the freespace wavenumber, and $d$ is the cavity depth of the liner.

For each of these liner configurations, Eq. (1) is used as the wall impedance boundary condition with a propagation code (also based on the convected Helmholtz equation) to simulate acoustic pressures (SPL and phase) at axial locations corresponding to the microphone locations in the GFIT. These simulated data are used to determine the number of microphones, chosen from those available for usage in the GFIT, required to achieve acceptable impedance eductions. 


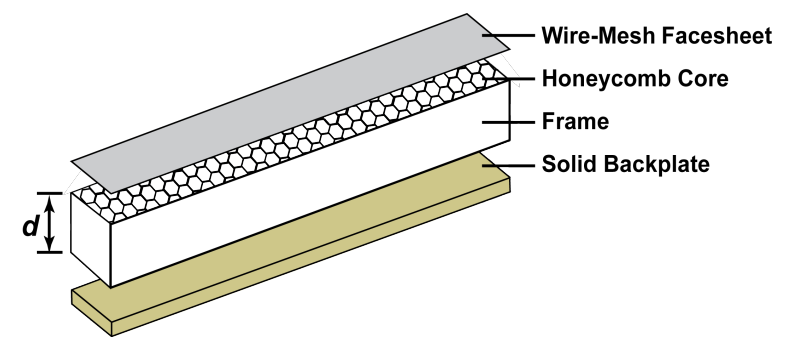

Figure 2: Sketch of wire mesh-over-honeycomb liner.

\section{Test Liners - Measured Data}

Two different liners are considered for the second portion of this study (see Fig. 3). The first is a ceramic tubular liner $(\mathrm{CT})$ that consists of parallel, cylindrical channels embedded in a ceramic matrix. These 0.6 mm-diameter channels are perpendicular to the exposed surface and provide a surface porosity of $57 \%$. The $85.6 \mathrm{~mm}$-deep channels are rigidly terminated such that each is isolated from its neighbor to ensure a locally-reacting structure. The channel diameter is small enough that SPL and grazing flow effects are insignificant relative to internal viscous losses. This liner provides an impedance spectrum that varies over a range typically observed in aircraft engine nacelle liners.

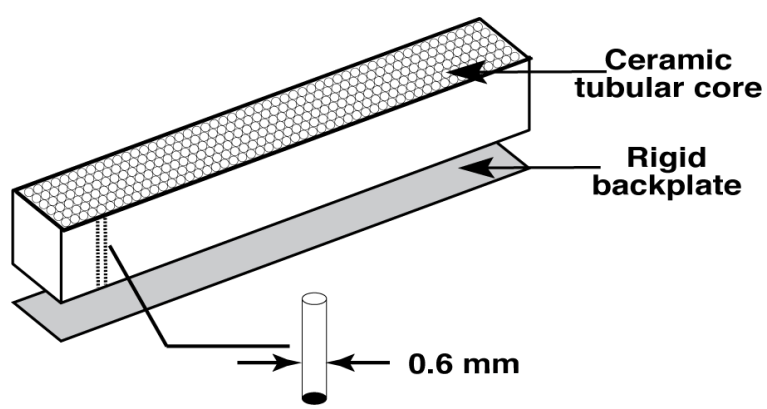

(a) Ceramic tubular liner.

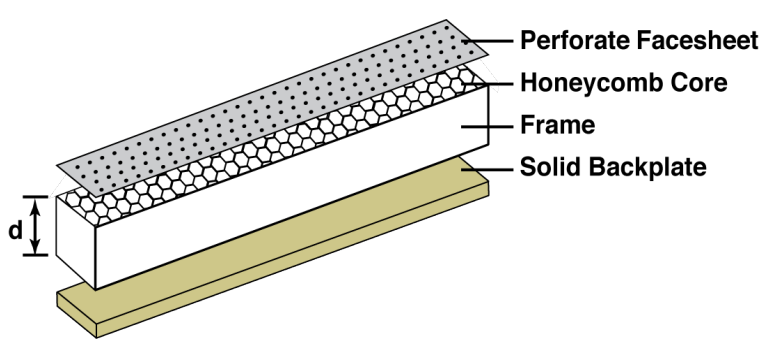

(b) Perforate-over-honeycomb liner.

Figure 3: Sketch of ceramic tubular and perforate-over-honeycomb liners.

The second is a conventional liner that consists of a single-layer, perforate-over-honeycomb (PH) representative of liners commonly used in current aircraft engine nacelles. This liner has a facesheet with $0.991 \mathrm{~mm}$-diameter holes and a thickness of $0.635 \mathrm{~mm}$, is bonded to a $38.18 \mathrm{~mm}$-deep cavity consisting of $9.5 \mathrm{~mm}$-diameter hexcel honeycomb core, and is terminated with a rigid backplate. It has a surface porosity of $8.7 \%$, and is nonlinear with respect to mean flow velocity and SPL.

\section{Impedance Eduction Methods}

Two impedance eduction methods are evaluated in this study. The first, labeled the CHE method, is based on a finite element solution of the convected Helmholtz equation. This method uses an estimate of the liner impedance as input to a duct acoustics propagation code that predicts the SPL and phase profiles over the length of the GFIT test window (see Fig. 1). The difference between measured and predicted SPL and phase profiles is evaluated. If the difference between the predicted and measured SPL and phase profiles is within an acceptable tolerance, the estimate for the impedance is taken as the unknown impedance of the liner. Otherwise, the impedance estimate is updated and the process is repeated iteratively until an impedance is found for which the difference is zero within an acceptable tolerance. The second impedance eduction method, labeled the KT algorithm, extracts the axial propagation constant from the measured SPL 
and phase profiles. This allows direct computation of the impedance of the acoustic liner by substituting this measured axial propagation constant and the normal mode solution to the convected Helmholtz equation into the wall impedance boundary condition.

\section{A. Convected Helmholtz Equation (CHE) Method}

A description of the CHE method is provided in an earlier paper by Watson, ${ }^{4}$ and is briefly summarized as follows. If the mean flow through the duct is assumed to be uniform, and only plane waves are assumed to be present in the spanwise direction (between the opposing hard walls), the acoustic field propagating through the flow duct (assuming a time dependence of the form $e^{i \omega t}$ ) satisfies a convected Helmholtz equation on the acoustic pressure field

$$
\left(1-M^{2}\right) \frac{\partial^{2} p(x, y)}{\partial x^{2}}+\frac{\partial^{2} p(x, y)}{\partial y^{2}}-2 i k M \frac{\partial p(x, y)}{\partial x}+k^{2} p(x, y)=0
$$

where $p$ is the acoustic pressure field, $i=\sqrt{-1}$ is the unit imaginary number, $k=\frac{\omega}{c}$ is the freespace wavenumber, $\omega$ is the angular frequency, $c$ is the speed of sound in the duct, and $M$ is the uniform flow Mach number. The normal component of the acoustic particle velocity vanishes at the rigid lower wall, which is equivalent to

$$
\frac{\partial p(x, 0)}{\partial y}=0
$$

Source and exit plane boundary conditions are given by the measured acoustic pressures,

$$
p(0, y)=p(0,0) ; p(L, y)=p(L, 0)
$$

When written in terms of the acoustic pressure field, the locally-reacting wall boundary condition ${ }^{20}$ is given by

$$
-\frac{\partial p(x, H)}{\partial y}=i k\left(\frac{p(x, H)}{\zeta}\right)+2 M \frac{\partial}{\partial x}\left(\frac{p(x, H)}{\zeta}\right)+\frac{M^{2}}{i k} \frac{\partial^{2}}{\partial x^{2}}\left(\frac{p(x, H)}{\zeta}\right)
$$

where the normalized admittance, $\frac{1}{\zeta}$, is taken as zero along the rigid wall portion of the upper wall. The CHE method of impedance eduction solves the boundary value problem defined in Eqs. (2)-(5) using a finite element method (FEM). The impedance boundary conditions are incorporated in the FEM analysis in weak form and cubic Hermite polynomials are used as the basis functions.

The unknown normalized impedance, $\zeta$, is educed by minimizing the objective function

$$
F(\theta, \chi)=\sum_{I=1}^{N}\left\|p\left(x_{I}, 0\right)_{\text {num }}-p\left(x_{I}, 0\right)_{\text {meas }}\right\|
$$

where $N$ is the number of microphones and $x_{I}$ is the location of the $I^{\text {th }}$ microphone. Throughout this paper, all impedances are normalized by $\rho c$, where $\rho$ is the density of air in the duct. The minimization is achieved using Stewart's adaptation of the Davidon-Fletcher-Powell (SDFP) optimization algorithm. ${ }^{21}$ SDFP returns the normalized resistance, $\theta$, and normalized reactance, $\chi$, of the test liner $(\zeta=\theta+i \chi)$.

\section{B. Kumaresan and Tufts (KT) Algorithm}

Application of the KT algorithm to liner impedance eduction has been described in an earlier paper. ${ }^{19}$ Therefore, only enough detail is presented in this paper to motivate the methods and goals of the current investigation. To begin, the acoustic pressure field in the liner test section $\left(L_{1} \leq x \leq L_{2}\right)$ of Fig. 1 can be expanded into a series of normal duct modes

$$
p(x, y)=\sum_{n=1}^{R} A_{n} P_{n}(y) e^{-i K_{n} x}, \quad P_{n}(y)=\cos \left(\lambda_{n} y\right), \quad \lambda_{n}^{2}=\left(k-K_{n} M\right)^{2}-K_{n}^{2}
$$


where $n$ is the mode order, $R$ is the total number of duct modes in the expansion, $A_{n}$ is the complex mode coefficient, $P_{n}(y)$ is the normal acoustic pressure mode, and $K_{n}$ is the axial propagation constant. Each term in the mode expansion [i.e., $A_{n} P_{n}(y) e^{-i K_{n} x}$ ] satisfies the convected Helmholtz equation [see Eq. (2)] and the lower wall boundary condition [Eq. (3)]. Substituting the mode solution into the wall impedance boundary condition [Eq. (5)] gives an expression for the impedance

$$
\zeta=\frac{i k}{\lambda_{n}}\left(1-K_{n} M / k\right)^{2} \cot \left(\lambda_{n} H\right)
$$

Note that if $K_{n}$ is measured using the lower wall microphone data (see Fig. 1), then Eq. (8) can be used to compute the unknown impedance, $\zeta$, of the test liner. Because each normal mode, $n$, gives the same impedance, the most accurately measured axial propagation constant, $K_{n}$, produces the most accurate impedance.

A couple of additional comments concerning the chosen number of modes, $R$, are necessary. The best solution is obtained when $R$ equals the number of modes in the measured signal. However, the number of modes cannot be known a priori. Further, if $R$ is chosen too small, there will be mode aliasing and the extracted axial propagation constants, $K_{n}$, will be perturbed from their true value. The best approach is therefore to choose $R$ greater than the number of modes in the signal. Choosing $R$ too large, however, has the disadvantage that extraneous modes may be generated. ${ }^{19}$ If $R$ is chosen larger than the number of normal modes in the duct, then a method of identifying the extraneous modes is needed. This can be accomplish via the $\mathrm{KT}$ algorithm, which is described briefly below.

1. Measure the acoustic pressure field at $N$ equally spaced points, $x=x_{1}, x=x_{2}, \ldots x_{N}$, on the lower wall of the flow duct.

2. From these acoustic pressure measurements, construct the prediction equations

$$
[C]=\left[\begin{array}{ccccc}
p_{2} & p_{3} & p_{4} & \cdots & p_{R+1} \\
p_{3} & p_{4} & p_{5} & \cdots & p_{R+2} \\
p_{4} & p_{5} & p_{6} & \cdots & p_{R+3} \\
p_{5} & p_{6} & p_{7} & \cdots & p_{R+4} \\
\vdots & \vdots & \vdots & \vdots & \vdots \\
p_{N-R+1} & p_{N-R+2} & p_{N-R+3} & \cdots & p_{N}
\end{array}\right],\{D\}=\left\{\begin{array}{c}
D_{2} \\
D_{3} \\
D_{4} \\
\vdots \\
D_{R+1}
\end{array}\right\},\{G\}=\left\{\begin{array}{c}
p_{1} \\
p_{2} \\
p_{3} \\
\vdots \\
p_{R}
\end{array}\right\}
$$

where $p_{1}, p_{2}, \ldots p_{N}$, are the measured values of $p(x, 0)$ at $x=x_{1}, x=x_{2}, \ldots x=x_{N}$, respectively. These are obtained from the microphone measurements. Note that the coefficients in the Hankel matrix, $[C]$, and right-hand-side vector, $\{G\}$, are known from the observed data set.

3. Use singular value decomposition to obtain the generalized inverse of $[C]$

$$
[C]^{-1}=[V][\Lambda]^{-1}\left[U^{*}\right]^{T}
$$

Here, the superscripts $T,-1$, and $*$ denote a matrix transpose, the generalized matrix inverse, and the complex conjugate, respectively. Further, $[U],[V],[\Lambda]$ are the matrix of left eigenvalues, matrix of right eigenvalues, and matrix of singular values, respectively. The singular value decomposition will allow the normal modes and the spurious modes to be separated.

4. The generalized matrix inverse is used to obtain the vector of polynomial coefficients, $\{D\}$

$$
\{D\}=-[C]^{-1}\{G\}
$$

5. The linear prediction coefficients $D_{2}, D_{3}, \ldots D_{R+1}$ from the solution vector $\{D\}$ are used to construct the $R^{\text {th }}$ degree polynomial, $F(X)$

$$
F(X)=1+D_{2} X+D_{3} X^{2}+D_{4} X^{3}+\ldots D_{R+1} X^{R}
$$

It can be shown that the zeroes of $F(X)$ are related to the axial propagation constants, $K_{n}$, in the mode expansion in Eq. (8). 
6. Use a complex polynomial root finder to find the zeroes, $X$, of the polynomial defined in Eq. (13).

7. Find the measured axial wave number, $K_{n}$, from the poles, $S$, of $F(X)$

$$
S=\frac{1}{X}=e^{i \Delta x K_{n}}=\|S\| e^{i \Psi}
$$

where $\Delta x$ is the increment spacing between the $N$ evenly spaced points, \|\| denotes the complex absolute value, and $\Psi$ is the phase angle of the pole. The axial wave numbers corresponding to poles with a magnitude inside the unit circle (i.e., $\|S\|<1$ ) are spurious while the remaining axial wave numbers (i.e., those for which $\|S\| \geq 1$ ) correspond to normal modes in the duct.

8. An acoustic impedance is computed for each normal mode using Eq. (8), and a Thompson-tau outlier rejection method is applied to remove outlier impedances.

\section{Results and Discussion}

Independent analyses are used to determine the optimal microphone locations for the indirect (CHE) and direct (KT) impedance eduction methods. The results are then combined such that the final set of microphones is the union of the two optimized sets. For clarity, those sets of microphone locations used with the CHE method will be denoted with a 'C' prefix, and those used with the KT algorithm will be denoted with a ' $\mathrm{K}$ ' prefix.

\section{A. CHE Method Results}

\section{Optimization process}

The process used for optimization of the microphone locations for use with the CHE method is summarized as follows. First, a simple model [Eq. (1)] is used to generate predicted impedance spectra, $\zeta_{\text {pred }}(f)$, for the TL1 and TL2 liners and the no-flow condition. This impedance is used as input to a duct acoustics propagation code based on the convected Helmholtz equation (CHE) to generate sound pressure levels $[\operatorname{SPL}(f, x)]$ and phases $[\phi(f, x)]$ at 53 axial locations over the range of $x \in\{0, L\}$ that correspond to the microphone locations currently employed. Random jitter $\left( \pm 0.5 \mathrm{~dB}, \pm 1.0^{\circ}\right)$, commensurate with the maximum error expected for the microphones used in the GFIT, is then applied to these acoustic pressure profiles to simulate the effects of measurement error. This randomization process is applied independently at each microphone location, i.e., a uniform-distribution random number generator is used to select a value for the variation in SPL and phase within the chosen randomization range. This process is performed 11 times (different random number distribution each time) to generate 11 unique acoustic pressure profiles $\left(\operatorname{SPL}_{j}(f, x)\right.$ and $\phi_{j}(f, x)$, where $j$ represents the $j^{\text {th }}$ data set).

The CHE method is then used to educe the impedance spectra $\left[\zeta_{j}(f)\right]$ for each of the 11 sets of data. Ideally, the educed impedance should identically match the predicted impedance if no random error is introduced, since the impedance eduction is performed with the same propagation code used to generate the acoustic pressure data. However, since random jitter corresponding to the GFIT measurement error is added to the predicted SPL and phase profiles, this is not the case. These 11 impedance spectra are used to compute the uncertainty limits ${ }^{22}$ (95\% confidence interval) of the educed impedance spectra.

Next, data corresponding to selected microphone locations are systematically removed from the 11 data sets, and the impedance eduction process is repeated, i.e., 11 new impedance spectra $\left[\zeta_{j}(f)\right]$ are educed. The mean of the impedance spectrum computed from these 11 educed impedance spectra is compared with the results achieved with the full set of 53 microphones. For those cases where this mean spectrum falls within the uncertainty limits computed from the original data, the reduced microphone set is deemed sufficient. Conversely, for those cases where the mean spectrum (or a portion thereof) falls outside the previously determined uncertainty limits, the reduced microphone set is deemed unacceptable. This process is used to determine the optimum subset of microphones. 


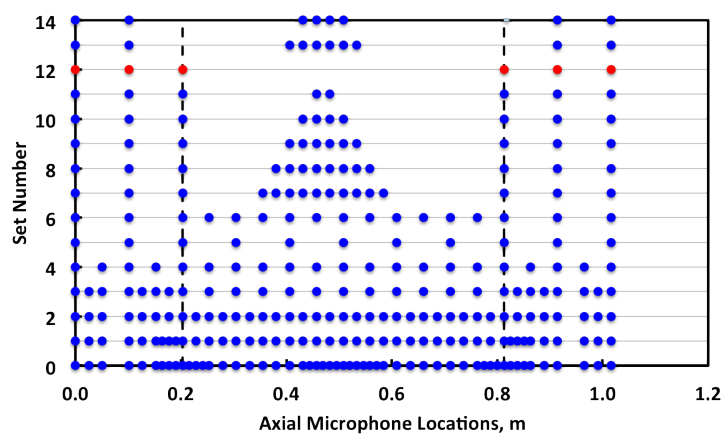

Figure 4: Depiction of microphone locations used in the CHE portion of this study. Set C0 (labeled as '0') is the baseline set. Symbols: blue $=$ pass, red = fail; dashed lines correspond to leading and trailing edges of liner.

\begin{tabular}{|c|c|c|c|}
\hline Set \# & \# mics & Description & Pass/Fail \\
\hline \hline C14 & 8 & Eliminated first and last mics in liner section & Pass \\
\hline C13 & 10 & Eliminated mics at LE and TE, added 6 mics in liner section & Pass \\
\hline C12 & 6 & Eliminated both mics in liner section & Fail \\
\hline C11 & 8 & Eliminated first and last mics in liner section & Pass \\
\hline C10 & 10 & Eliminated first and last mics in liner section & Pass \\
\hline C9 & 12 & Eliminated first and last mics in liner section & Pass \\
\hline C8 & 14 & Eliminated first and last mics in liner section & Pass \\
\hline C7 & 16 & Repositioned 10 mics in liner section, away from liner LE and TE & Pass \\
\hline C6 & 17 & Added mics in liner section (to enhance KT usage) & Pass \\
\hline C5 & 11 & Eliminated half of mics & Pass \\
\hline C4 & 21 & Eliminated half of mics in hard wall sections & Pass \\
\hline C3 & 27 & Eliminated half of mics in liner section & Pass \\
\hline C2 & 39 & Eliminated mics in hard wall sections at non-integral locations & Pass \\
\hline C1 & 43 & Eliminated mics in liner section not suitable for KT algorithm & Pass \\
\hline C0 & 53 & Full set of mic locations available in GFIT lower wall & Pass \\
\hline
\end{tabular}

Table 1: Microphone locations used for optimization study.

Figure 4 provides a graphical depiction of the 15 sets of microphone locations used in this analysis. Blue circles correspond to axial locations of microphones for data sets deemed to be sufficient ('pass') for quality impedance eduction. Red circles correspond to axial locations for the subset of microphone locations determined to be insufficient ('fail') for quality impedance eduction (only occurs for Set C12). Set C0 corresponds to the original set of 53 microphone locations (i.e., a typical, full set of measurement data). Table 1 provides descriptions for each of the 15 microphone distributions. For the sake of brevity, the descriptions for each microphone distribution are relative to their respective predecessors, i.e., using a building block approach.

Given the amount of time required for this impedance eduction approach, an exhaustive review of all possible microphone combinations was outside the scope of this study. Instead, a number of the microphone distributions were selected because of their perceived suitability for the KT algorithm. The first sets (Sets C1 to C6) of microphone distributions were selected because of their perceived 'dual-use' value, i.e., they were expected to be suitable for both the CHE and KT impedance eduction methods. Microphone locations over 
the liner section were then redistributed (Sets C7 to C12) to (1) move the interior microphones away from the leading and trailing edges of the liner to minimize the effects of reflections, and (2) reduce the number of microphones over the liner section by as much as possible. The last of this group was Set C12, for which all microphones over the liner section were removed from the data sets. As described below, this microphone distribution was shown to be unacceptable. The last two microphone distributions (Sets C13 and C14) examined the need for including microphones at the leading and trailing edges of the liner. It should be noted that, due to the limitations on the number of microphone sets that could be evaluated, it is entirely possible that there exists another microphone array that would provide slightly better results. Nevertheless, the results achieved with the current microphone sets are believed to be sufficient for the purposes of this study.

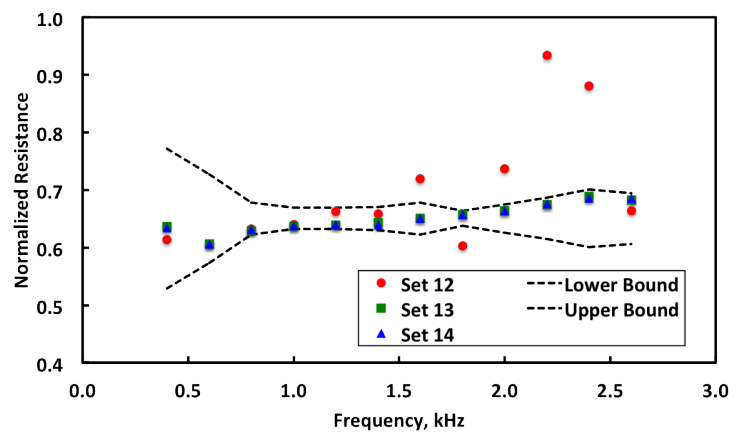

(a) Normalized Resistance

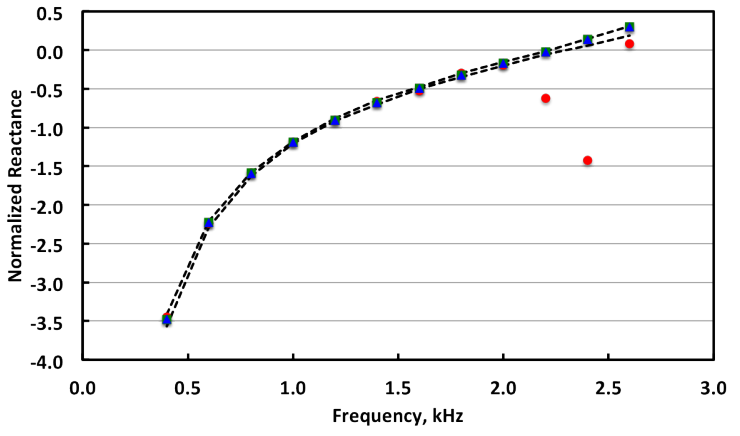

(b) Normalized Reactance

Figure 5: Comparison of impedance spectra educed with the CHE method for the TL1 liner. Dashed lines provide bounds of $95 \%$ confidence interval achieved using 53 microphone locations (Set C0), and symbols provide mean values with 6 (red circles; Set C12), 10 (green squares; Set C13), and 8 (blue triangles; Set C14) microphone locations, respectively.

Figure 5 provides a graphical depiction of the optimization process as it applies to the last three sets of microphone locations included in the optimization study (Sets C12, C13, and C14). The results provided in this figure correspond to analysis conducted with the TL1 liner. The corresponding results for the TL2 liner provided very similar results, and are therefore not shown for the sake of brevity. The dataset containing 53 microphones (Set C0 in Fig. 4) is used to determine the acceptable error bounds, where the bias error is taken to be the difference between the mean impedance and the true impedance at each frequency, and random error is given by the $95 \%$ confidence interval of the 53 microphone set. The $95 \%$ confidence interval is computed from impedance spectra educed for 11 sets of simulated data created by applying random jitter to the acoustic pressures that corresponded to the 53 GFIT microphone locations typically used for impedance eduction with the CHE method.

The confidence interval for the resistance component is provided in Fig. 5-a. The interval has a fairly constant magnitude over the middle portion of the frequency regime, and is larger at the two frequency extremes. This is likely due to the minimal amount of attenuation provided by this liner at the lower and upper frequency extremes, which causes the impedance eduction process to be somewhat less robust (larger confidence interval). The reactance component is shown in Fig. 5-b. Due to the strong $-\cot (k d)$ nature of the reactance component, the spread between the lower and upper limits of the $95 \%$ confidence interval is difficult to visualize. Regardless, the same process as is shown for the resistance component was also applied for the reactance component. Finally, recall that the CHE propagation code assumes a plane wave is present at the source and exit planes of the computational domain. While it is acceptable to use the CHE code to simulate data that abide by this constraint, the dimensions of the GFIT are such that a higher-order mode cuts on at a frequency between $2.6 \mathrm{kHz}$ and $2.8 \mathrm{kHz}$. Since it is not typically known a priori whether this higher-order mode contains sufficient energy to affect the results, the CHE is not used for impedance eduction in this frequency range. For this reason, the results presented for this portion of the study are limited to frequencies up to $2.6 \mathrm{kHz}$. 
The symbols in Fig. 5 correspond to the mean values of impedance at each frequency. The data for Set $\mathrm{C} 12$ are used to describe the process. Recall that each of the 11 sets of simulated data noted above had a different distribution of random jitter applied to the simulated acoustic pressure at each microphone location. The data at all but the 6 microphone locations indicated in Fig. 4 and Table 1 were removed from each of these data files, and the resultant data were used as input to the CHE impedance eduction method. This resulted in 11 educed impedance spectra. The mean values of these 11 impedance spectra are presented as red circles in Fig. 5. Clearly, the values at 5 of the frequencies fall outside the confidence interval limits, thereby indicating that this is an insufficient data set for use in the impedance eduction process. This is not at all surprising, as this microphone distribution contains no microphones in the liner section.

The other two data sets (Sets C13 and C14) shown in Fig. 5 are completely (all frequencies) encompassed by the $95 \%$ confidence interval. Hence, these two microphone distributions are deemed sufficient for quality impedance eduction using the CHE method. It should be noted that, although the results for the remainder of the microphone distributions (Sets $\mathrm{C} 1$ through C11) are not shown, the impedances educed with each of these distributions were also within the bounds of the $95 \%$ confidence interval.

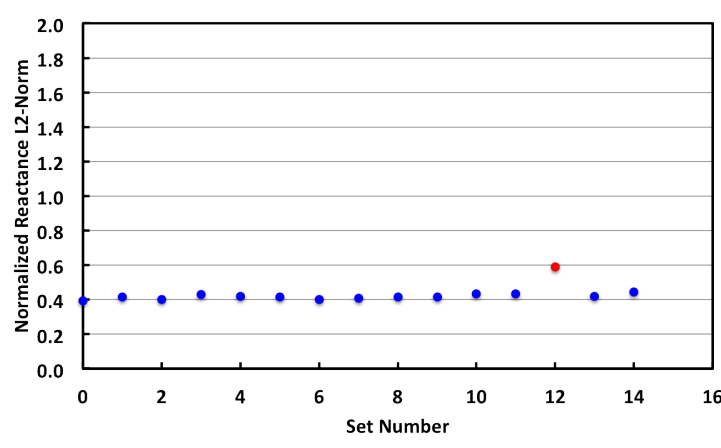

(a) $L_{2}$-Norm for normalized resistance.

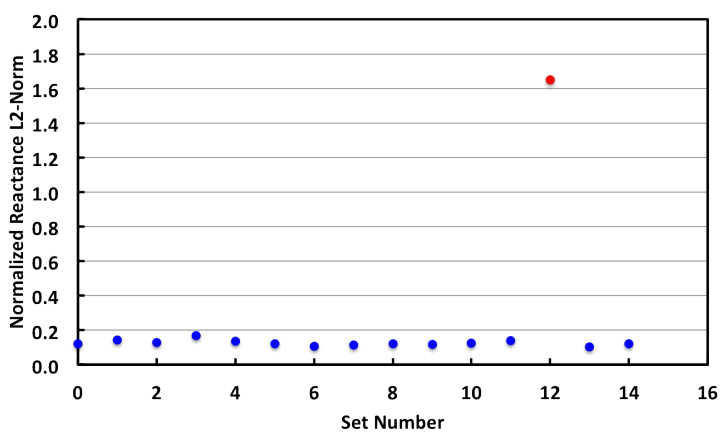

(b) $L_{2}$-Norm for normalized reactance.

Figure 6: $L_{2}$-Norm for normalized impedance results educed with TL1 liner using the CHE method. Set C12 is highlighted with a different color to indicate that the $L_{2}$-Norm for this set is unacceptably high.

Figure 6 gathers the results for the 15 microphone distributions considered in this study (Sets $\mathrm{C} 0$ to C14). The $L_{2}$-Norms given by

$$
L_{2, \text { res }}=\left(\sum_{f_{\min }}^{f_{\max }}\left(\theta_{s}-\theta_{\text {true }}\right)^{2}\right)^{0.5} ; \quad L_{2, \text { rea }}=\left(\sum_{f_{\min }}^{f_{\max }}\left(\chi_{s}-\chi_{\text {true }}\right)^{2}\right)^{0.5}
$$

are computed for the normalized resistance (Fig. 6-a) and reactance (Fig. 6-b), where $\theta_{s}$ and $\chi_{s}$ correspond to the values for normalized resistance and reactance educed with the selected set of microphones, $\theta_{\text {true }}$ and $\chi_{\text {true }}$ correspond to the true values (i.e., the values used as input for the initial simulation) for normalized resistance and reactance, and $f_{\min }$ and $f_{\max }$ correspond to the minimum and maximum frequencies $(0.4$ and $2.6 \mathrm{kHz}$ ) used in this evaluation.

The $L_{2}$-Norms for the resistance, $\theta$, and reactance, $\chi$, components have values of 0.39 and 0.12 , respectively, for the baseline (Set $\mathrm{C} 0$ ) microphone set that uses all 53 microphones. According to Eq. (15), the deviation of these values from zero indicates a bias error in the original (CHE) impedance eduction process, at least for the current liner and test conditions. The variability away from these two values for the $L_{2}$-Norm represents additional error, whether bias or random, due to changes in which microphones are used in the analysis. It is this latter source of error that is the subject of the current investigation. For the remainder of the microphone sets, the results are consistent with those described above, i.e., all but Set C12 provide very similar $L_{2}$-Norms, thus indicating they are all relatively equally acceptable for use with the CHE impedance eduction method. In summary, the only microphone distribution that was found to be insufficient was the 6-microphone distribution labeled as Set C12. 


\section{Validation process}

The process described above contains two clear limitations: (1) only one liner type (wire mesh-over-honeycomb) is included in the evaluation, and (2) the effects of flow, which can have a significant effect on the liner impedance, are neglected. These items were addressed as follows. Acoustic pressure data were acquired in the GFIT (all 53 microphones along the wall opposite the liner) for the ceramic tubular and perforate-overhoneycomb liners, at centerline Mach numbers of 0.0, 0.3, and 0.5. For each test liner and test condition, two impedance eductions were conducted using the CHE method. The first employed the acoustic pressure data from all 53 microphones, whereas the second employed only those acoustic pressures for the subset of microphones determined via the optimization process described above. As shown in Tab. 1, the number of microphones used in the second eduction ranged from 6 (Set C12) to 43 (Set C2).

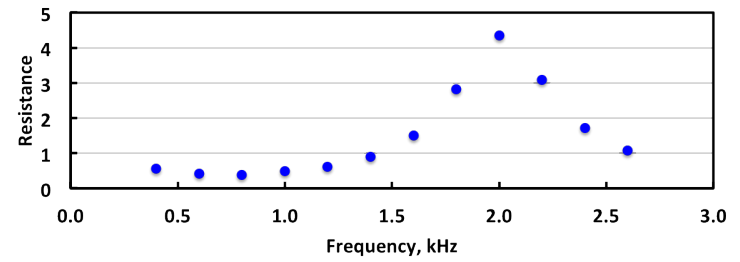

(a) Results for Set C0; CT liner.

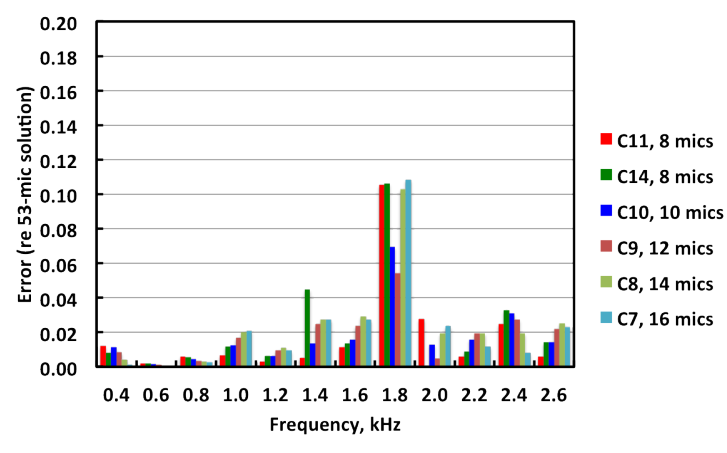

(c) Error spectrum; CT liner.

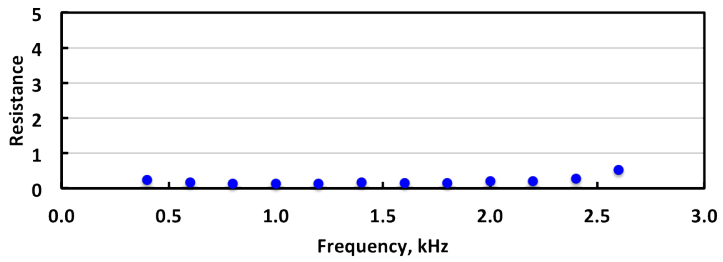

(b) Results for Set C0; PH liner.

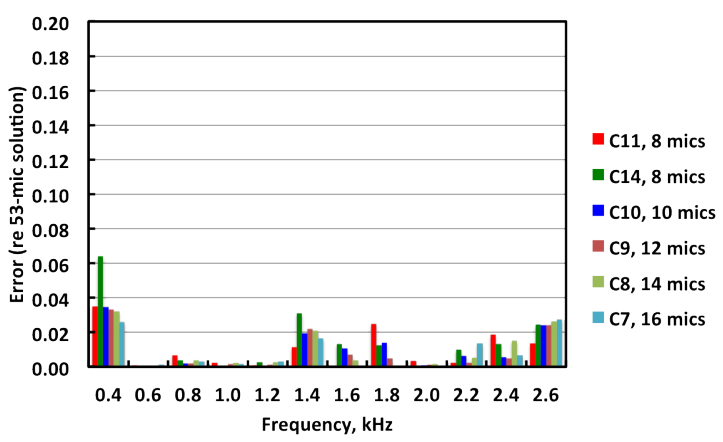

(d) Error spectrum; PH liner.

Figure 7: Normalized resistance and resistance errors relative to values educed using the full set of 53 microphones for ceramic tubular (CT) and perforate-over-honeycomb (PH) liners; Mach 0.0.

Figures 7 and 8 provide normalized resistance spectra educed with the ceramic tubular (CT) and perforate-over-honeycomb $(\mathrm{PH})$ liners at the Mach 0.0 and 0.3 conditions. They also provide error results for the normalized resistance spectra. In the error plots, six bars corresponding to results achieved with six microphone distributions are provided at each frequency of interest. These distributions are chosen because they require the fewest number of microphones, and were all deemed acceptable based on the optimization study described above. Note that two of the data sets (Sets C11 and C14) contain 8 microphones, one that includes microphones at the liner leading and trailing edges, and one that does not.

The error values, $E_{s}$, assigned to each data set at a selected frequency are computed as

$$
E_{s}=\left|\theta_{s}-\theta_{53}\right|
$$

where $\theta_{53}$ corresponds to the normalized resistance educed with the full set of 53 microphones. Similar computations were also conducted with the reactance component, and were found to be consistent with the observations discussed below for the resistance component.

Looking first at Fig. 7, the error values are observed to be quite small, typically less than 0.04 units (recall that all impedances are normalized with respect to the quantity $\rho c$, where $\rho$ and $c$ correspond to the density and sound speed of air at ambient conditions, respectively) for both liners (CT and PH). A noticeable increase in error is observed at $1.8 \mathrm{kHz}$ for the $\mathrm{CT}$ liner. Comparison with the corresponding normalized 
resistance spectrum suggests that this increase in error is associated with the significant increase in resistance that occurs near this frequency. It should also be noted that this frequency is near an anti-resonance for this liner. To a lesser extent, the error increases at $0.4 \mathrm{kHz}$ for the $\mathrm{PH}$ liner. This is likely due to the fact that this liners is very inefficient at sound absorption at this frequencies. At $1.4 \mathrm{kHz}$, the ' $\mathrm{C} 14$ ' error for the CT liner is observed to be somewhat larger than that achieved with the other microphone distributions (including the other configuration with only 8 microphones). Finally, the errors are observed to increase for the $\mathrm{PH}$ liner at the mid-frequencies (1.4 to $1.8 \mathrm{kHz}$ ), where large attenuations occur.

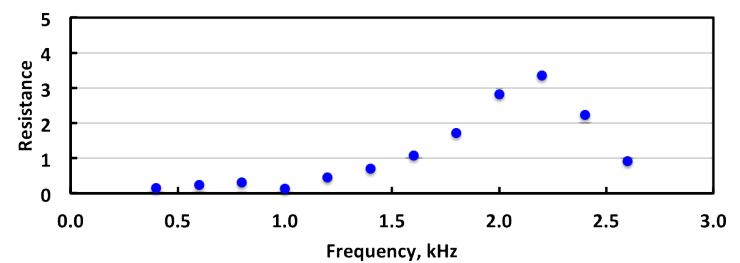

(a) Results for Set C0; CT liner.

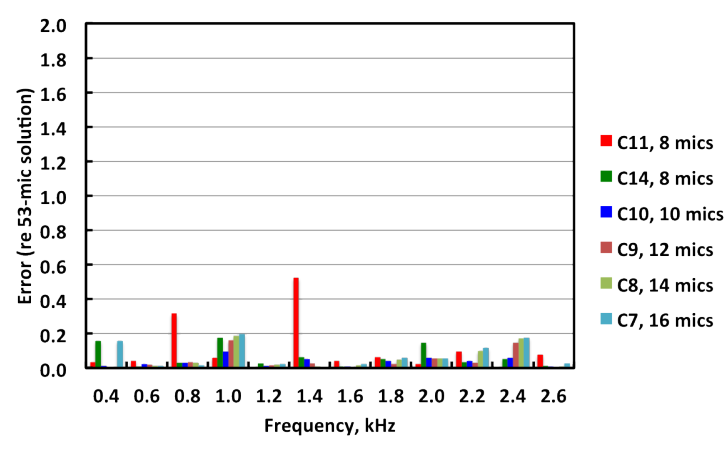

(c) Error spectrum; CT liner.

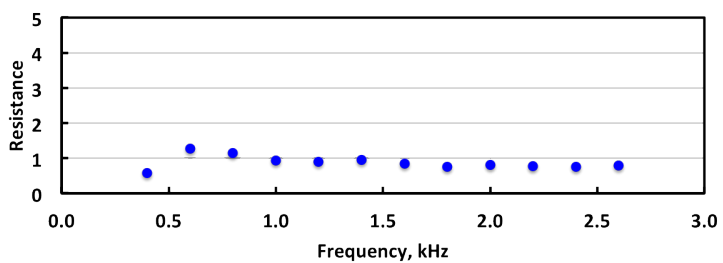

(b) Results for Set C0; PH liner.

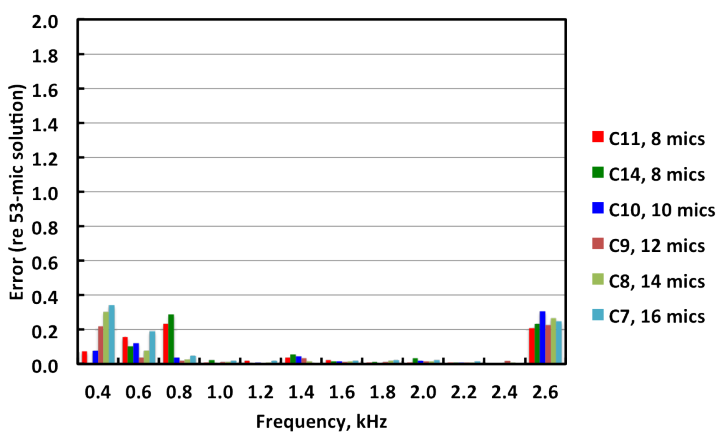

(d) Error spectrum; PH liner.

Figure 8: Normalized resistance and resistance errors relative to values educed using the full set of 53 microphones for ceramic tubular $(\mathrm{CT})$ and perforate-over-honeycomb (PH) liners; Mach 0.3.

The corresponding results for the Mach 0.3 case are provided in Fig. 8. There is minimal change in the resistance spectrum for the CT liner because it consists of a number of cylindrical tubes with a high length-to-diameter ratio. On the other hand, there is a significant increase in the resistance spectrum of the $\mathrm{PH}$ liner as the Mach number is increased to 0.3. For both liners, the errors are larger than observed for the no-flow condition. For the CT liner (Fig. 8-c), the largest errors occur for the first microphone distribution (Set C11) with 8 microphones, i.e., the set that includes microphones at the liner leading and trailing edges. For the PH liner (Fig. 8-d), the largest sources of error occur at the frequency extremes, where the reactance magnitude becomes large (i.e., at frequencies where minimal attenuation occur). Similar results are observed at Mach 0.5, and are therefore not shown for the sake of brevity.

Overall, these results suggest that the optimal set of microphones contains 14 or 16 microphones (Set C8 or Set C7). However, there is clearly significant latitude in the choice of microphones for successful implementation of the CHE impedance eduction method.

\section{B. KT Algorithm Results}

\section{Optimization process}

The process here very strongly mirrors the approach taken for the CHE method. Since the results of the TL1 and TL2 liners do not vary significantly for the CHE, it is not expected that there will be different behavior with the KT algorithm. Under this assumption, the optimization is performed only with the TL1 liner at the no-flow condition. The SPL and phase are obtained at the same 53 axial locations and random jitter is applied as described in Section IV.A.1. However, the reduced computational requirements 
of the KT algorithm allow for a Monte Carlo approach to be taken in determining the statistics of the educed impedances. To this end, a population of 20,000 pressure profiles is generated, which is sufficient for convergence of the standard deviation of the impedance spectrum.

Due to the limitations of the KT algorithm, the microphone locations are restricted to the evenly spaced microphones. The GFIT lower wall contains 33 microphones that are spaced $25.4 \mathrm{~mm}$ apart. However, four microphones are located upstream of the liner leading edge (LE) and another four are located downstream of the liner trailing edge (TE). These microphones are located in hard wall sections of the duct, and are therefore not suitable for use in the KT algorithm since there is no means to account for the impedance discontinuity at the LE and TE of the liner. Removing these microphones reduces the total usable number of microphones to 25. Rather than choosing individual sets of microphones and iterating on the process manually, the speed of the impedance eduction process allows for every possible configuration of these microphones to be tested. To that end, the total number of microphones is varied from 4 to 25. The microphones are also shifted along the length of the liner (e.g. for 24 microphones, two sets exist: microphones 1-24 and microphones 2-25), exhausting each possible combination of microphones. The impedance eduction process is carried out as described in Section III.B.

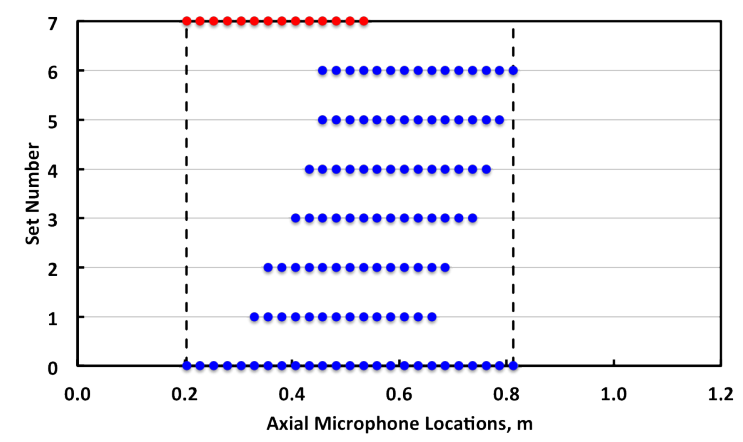

Figure 9: Depiction of microphone locations used in the KT method portion of the study. Set K0 is the baseline set. Symbols: blue = pass, red = fail; dashed lines correspond to leading and trailing edges of liner.

Because of the criteria used to determine the best impedance, the KT algorithm occasionally chose an impedance estimate that was an outlier when compared to the rest of the population. In all cases where this occurred, there was another choice of impedance that was within the expected distribution. This suggests that it is not sufficient to depend on the pole estimation used with the KT method, and that the criteria used in the optimization are also critical. While it may be reasonable to identify and correct this for a single realization of the impedance spectrum, this becomes prohibitive for the population size chosen here. Therefore, in order to ensure the results were representative only of the error in the method, a Thompson-tau outlier rejection method was applied to the impedance population. This is implemented by removing the samples that are more than three standard deviations from the mean and iterating until all of the outliers are removed. Each microphone set is then checked to ensure that the impedance spectrum is within reasonable limits.

The set containing 25 microphones (Set K0 in Fig. 9) is used as a baseline of the acceptable error bounds, where the bias error is taken to be the difference between the mean impedance and the true impedance at each frequency, and random error is given by the $95 \%$ confidence interval of the 25 microphone set. For a particular group of microphones to be accepted as suitable for impedance eduction, the mean impedance spectra must be within the range corresponding to the sum of the bias and random error present in the 25 microphone case set centered about the true impedance. The set of microphones that remains in the error bounds for the entire spectrum while minimizing the number of microphones is determined to be the optimum. In this case, the minimum number of microphones needed is 14. Sets K1-K6, diagrammed in Fig. 9, correspond to the shifted sets that are valid for either 14 or 15 microphones. 


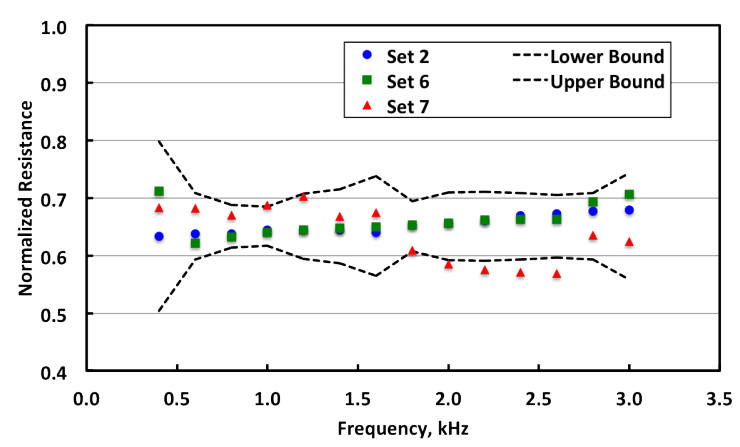

(a) Normalized Resistance

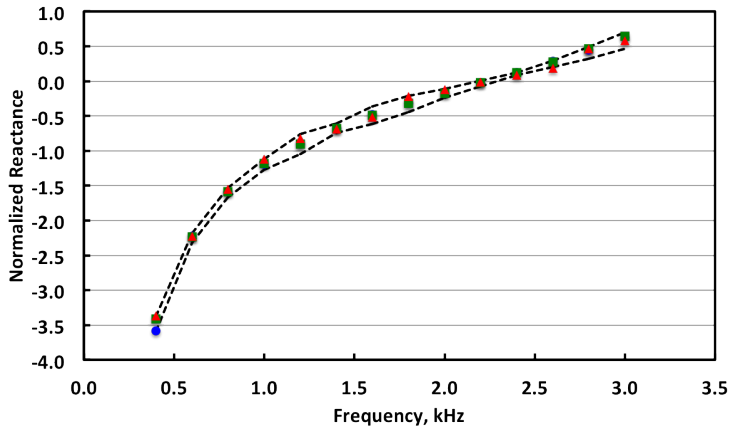

(b) Normalized Reactance

Figure 10: Comparison of impedance spectra educed with the KT method for the TL1 liner. Dashed lines provide bounds of $95 \%$ confidence interval achieved using 25 microphone locations (Set K0), and symbols provide mean values with 14 microphone locations placed near the middle (blue circles; Set K2), trailing edge (green squares; Set K6), and leading edge (red triangles; Set K7) of the liner, respectively.

A comparison of the impedance spectra educed with Sets K2, K6, and K7 is provided in Figure 10. Set K7 is provided as a comparison of accuracy for the same number of microphones placed at the front of the liner. Overall, it is found that placing microphones near the leading edge of the liner results in a larger loss of accuracy than placing them near the trailing edge. Even less error is observed for configurations using microphones away from the LE and TE of the liner. The minimum total error is achieved by Set K2, which is slightly biased to the rear of the liner (by a single microphone).

It can be seen that placing the microphones at the front of the liner (Set K7) causes a large portion of the impedance spectrum to be outside of the acceptable range, the majority of which are discarded due to poor estimation of the resistance. Apart from that, the results follow similar trends as seen with the CHE with respect to the spectral shape of the error bounds and mean impedances of the acceptable microphone combinations. The $L_{2}$-Norms (Eq. 15) for all of the sets are given in Fig. 11. This shows nearly a doubling of the $L_{2}$-Norm when the microphones are placed at the front of the liner as compared to positioning them at the center or rear of the liner. As was the case for the CHE, there is no one set that emerges as being more acceptable than any other.

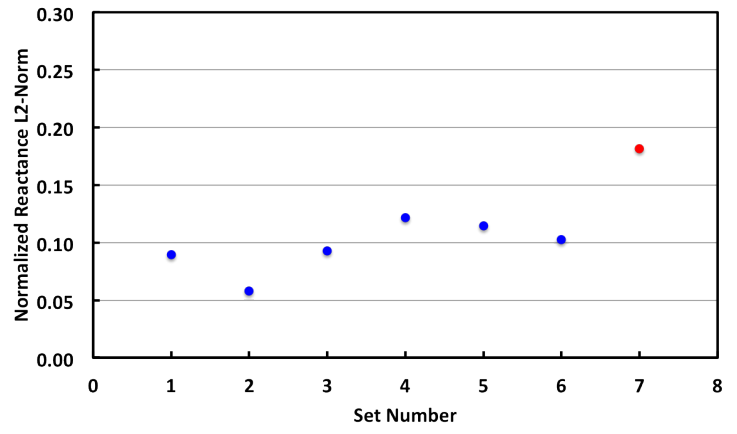

(a) $L_{2}$-Norm for normalized resistance.

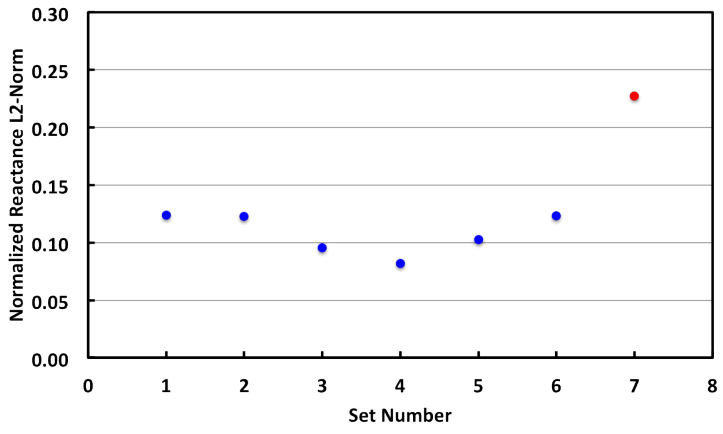

(b) $L_{2}$-Norm for normalized reactance.

Figure 11: $L_{2}$-Norm for normalized impedance results educed with TL1 liner using the KT method. Set K7 is highlighted with a different color to indicate that the $L_{2}$-Norm for this set is unacceptably high. 


\section{Validation process}

In an effort to show that the results of the optimization hold for a variety of liners, two microphone configurations are used to educe the impedance spectra of the CT and PH liners at the same three flow speeds as used to validate the CHE. Due to the reduced length of the CT and PH liners when compared to the TL1 liner, the errors are referenced to the same CHE case with 53 microphones, rather than a case of 16 microphones educed by the KT method. Figures 12 and 13 show the relative error between the CHE reference case and the two test cases. The first test case is a 'full' set of 14 microphones (Set K2 from Section IV.B.1), determined from the results of the previous section. However, the smaller liner places these microphones close to the leading edge of the liner. To investigate whether it is more beneficial to avoid the leading edge, a 'reduced' set is proposed that begins approximately $102 \mathrm{~mm}$ from the leading edge of the liner but contains only 10 microphones (removed first two and last two microphones from Set K2).

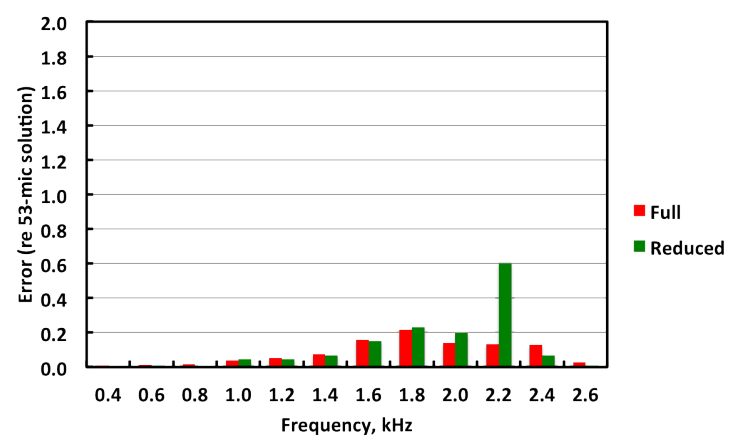

(a) Ceramic tubular (CT) liner.

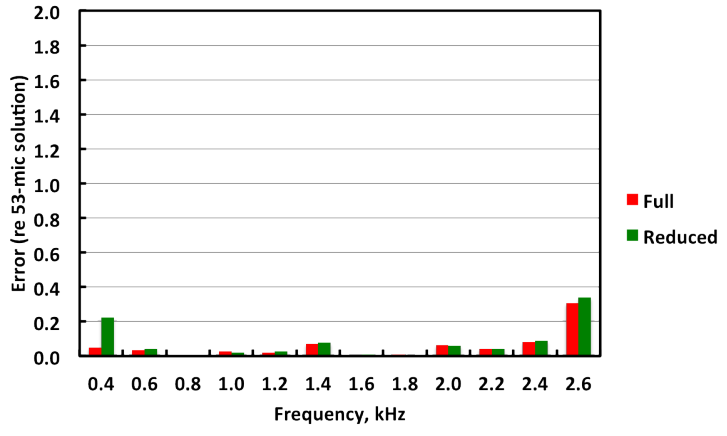

(b) Perforate-over-honeycomb (PH) liner.

Figure 12: Normalized resistance errors relative to values educed with CHE using the full set of 53 microphones; Mach 0.0.

Similar error trends to those seen in Figs. 7 and 8 are seen for the KT method, albeit at larger magnitudes. The accuracy of KT educed impedances suffers significantly near anti-resonance of the CT liner $(2 \mathrm{kHz})$ for both the no-flow and Mach 0.3 cases. As mentioned earlier, poor attenuation over the liner is typically tied to a reduction in accuracy. This can also be seen near the low and high frequency portions of the PH liner spectra. These results indicate that the reduced and full sets are nearly equivalent in terms of accuracy. In comparison to the CHE, the KT method produces slightly less accurate results overall.

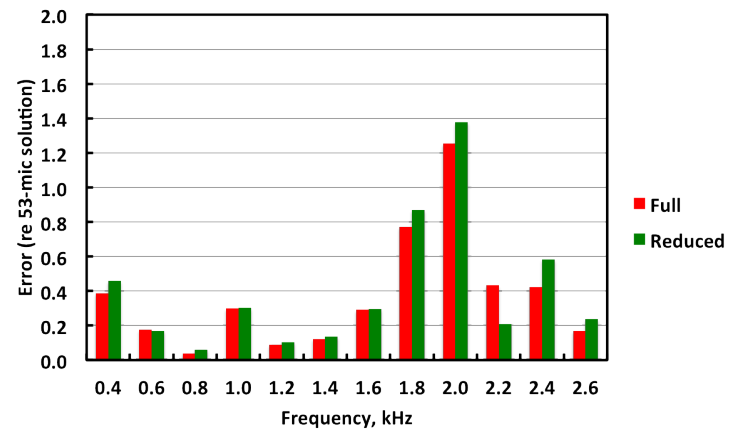

(a) Ceramic tubular (CT) liner.

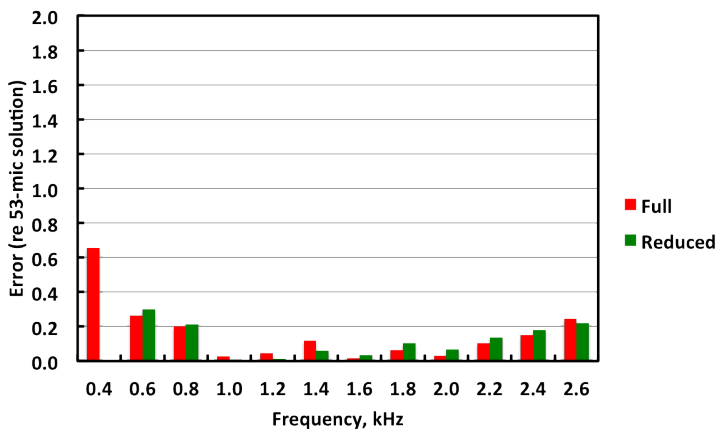

(b) Perforate-over-honeycomb (PH) liner.

Figure 13: Normalized resistance errors relative to values educed with CHE using the full set of 53 microphones; Mach 0.3. 


\section{Composite Microphone Set}

The last task of this study is to find a set of microphones that is suitable for use with both of the impedance eduction methods. Figure 14 provides a diagram of the microphone locations used for this final analysis. There is significant overlap between microphone Sets C7 and K2, each of which was shown to provide quality impedance eductions for the respective methods. Thus, the union of these two sets (Set CK1) would seem to be an appropriate choice for liners that are full length $(610 \mathrm{~mm})$. Since many of the liners built for testing in the GFIT are only $406 \mathrm{~mm}$ long, care should also be taken to ensure that the composite microphone set is suitable for liners of this length. Recall that the 'reduced' set used with the KT method was just a subset of Set K2. Therefore, any set of microphone locations that includes those from Set K2 should also be sufficient for evaluation of the shorter liners with the KT method.

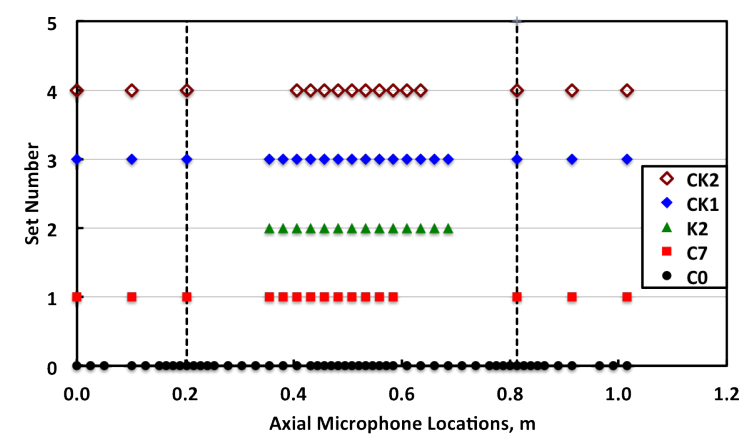

Figure 14: Depiction of full (Set C0), optimum CHE (Set C7), optimum KT (Set K2), full composite (Set CK1), and reduced composite (Set CK2) microphone locations.

The same process as was used with the CHE method to evaluate the initial 14 sets of microphone locations (Sets C1 through C14) was applied for this composite set (Set CK1). It was also applied to one additional set (Set CK2), for which four of the microphone locations of Set CK1 are eliminated. As expected, the results for both composite sets were observed to be within the uncertainty bounds. The corresponding $L_{2}$-Norms were also equal to or less than those of the other microphone sets evaluated with the CHE method.

Finally, the two composite sets of microphone locations were evaluated with data acquired in the GFIT for the CT and PH liners, at Mach numbers of 0.0, 0.3, and 0.5. As expected, the results were quite favorable. Hence, if $407 \mathrm{~mm}$-long liners are to be tested in the GFIT, an appropriate choice of microphone locations is given by Set CK2. This set contains 20 microphone locations. For $610 \mathrm{~mm}$-long liners, Set CK1 is preferred, with a total of 16 microphone locations. However, since Set CK2 is simply a subset of Set CK1, the convenient approach is to always acquire data using the Set CK1 microphone locations.

\section{Concluding Remarks}

This paper has explored two impedance eduction methods for use with data acquired in the NASA Langley Grazing Flow Impedance Tube (GFIT). The first method is an indirect method based on the convected Helmholtz equation (CHE), and the second method is a direct method based on the Kumaresan and Tufts algorithm (KT). Synthesized no-flow data based on two wire mesh-over-honeycomb liners were used for evaluation of a number of possible GFIT microphone configurations. These data were adjusted to include random jitter consistent with measurement error typically experienced in the GFIT.

This process was performed 11 times with the CHE impedance eduction method, and $95 \%$ confidence intervals were used to determine the acceptable bounds for impedances educed using this method. Next, a number of microphone locations were removed from the 11 sets of synthesized data (for each liner), and the CHE method was used to educe the impedance spectra for each dataset. A comparison of the mean impedance spectra and the $95 \%$ confidence interval determined from the original datasets (with all microphone locations included) was used to determine those sets of microphone locations for which acceptable impedances were educed. The CHE method was found to be quite robust. Indeed, a wide variety of microphone locations 
were found to be acceptable. Briefly, each array that included microphones in all three regions of the GFIT (upstream hard wall, liner, and downstream hard wall sections) was shown to provide acceptable results, even when the total number of microphones was reduced to eight. However, the best arrays were found to (1) include microphones in the upstream and downstream hard wall sections, and (2) include microphones opposite the liner, but well away from the leading and trailing edges of the liner.

Since the KT method requires far less computational resources, a Monte Carlo approach was used for this portion of the study. For this method, 20,000 sets of synthesized data were generated with random jitter, and were processed in the same manner as was used for the CHE method. The KT method cannot use the data acquired in the hard wall sections; thus, only the microphones opposite the liner were considered. A number of microphone arrays were considered that employed from 4 to 25 microphones. The results suggest that the optimum is to use 14 microphones positioned opposite the liner. Ideally, these microphones should be well away from the leading and trailing edges of the liner.

Each of the sets of microphone locations that were determined to be acceptable, whether with the CHE method or the KT method, was then evaluated with data measured in the GFIT. These measured data were acquired with a ceramic tubular liner and a perforate-over-honeycomb liner at three flow conditions (Mach 0.0, 0.3, and 0.5). The impedances educed with each eduction method (CHE or KT) using a reduced set of microphones were compared with impedances educed with the CHE method and the full set of 53 microphones. A favorable comparison for each of the liners and test condtions was used to demonstrate the suitability of each set of microphone locations.

Apart from this last validation with the ceramic tubular and perforate-over-honeycomb liners, the CHE and KT studies were conducted independently. As expected, a different set of optimum microphone locations was selected for each method. However, the respective microphone arrays were found to have a significant amount of overlap. Thus, the optimum set of microphone locations is determined to be the union of these two microphone arrays. This array contains 3 microphones in the upstream hard wall section, 14 microphones opposite the liner, and 3 microphones in the downstream hard wall section.

\section{Acknowledgments}

The Advanced Air Transport Technologies Project of NASA's Advanced Air Vehicles Program funded this work.

\section{References}

\footnotetext{
${ }^{1}$ Lockard, D. P. and Lilley, G. M., "The Airframe Noise Reduction Challenge," NASA TM 213013, 2004.

${ }^{2}$ Armstrong, D. L., Beckemeyer, R. J., and Olsen, R. F., "Impedance Measurements of Acoustic Duct Liners With Grazing Flow," Paper presented at 87th Meeting of the Acoustical Society of America 1999-1864, 1974.

${ }^{3}$ Parrott, T. L., Watson, W. R., and Jones, M. G., "Experimental Validation of a Two-Dimensional Shear-Flow Model for Determining Acoustic Impedance," NASA TP 2679, May 1987.

${ }^{4}$ Watson, W. R., Jones, M. G., and Parrott, T. L., "Validation of an Impedance Eduction Method in Flow," AIAA Journal, Vol. 37, No. 7, July 1999, pp. 818-824.

${ }^{5}$ Malmary, C., Carbonne, S., Auregan, Y., and Pagneux, V., "Acoustic Impedance Measurement With Grazing Flow," AIAA Paper 2001-2193, May 2001.

${ }^{6}$ Gallman, J. M. and Kunze, R. K., "Grazing Flow Acoustic Impedance Testing for the NASA AST Program," AIAA Paper 2002-2447, June 2002.

${ }^{7}$ Syed, A. A., Yu, J., Kwan, H. W., and Chien, E., "The Steady Flow Resistance of Perforated Sheet Materials in High Speed Grazing Flows," NASA CR 2002-211749, 2002.

${ }^{8}$ Jones, M. G., Watson, W. R., Tracy, M. B., and Parrott, T. L., "Comparison of Two Waveguide Methods for Educing Liner Impedances in Grazing Flow," AIAA Journal, Vol. 42, No. 2, Februrary 2004, pp. 232-240.

${ }^{9}$ Lavieille, M., Simon, F., and Micheli, F., "Measurement of Liner Acoustic Impedance in a Shear Layer of a Subsonic Flow by Laser Doppler Velocimetry," Sapem 2005, December 2005.

${ }^{10}$ Elnady, T., Boden, H., and Elhadidi, B., "Validation of an Inverse Semi-Analytical Technique to Educe Liner Impedance," AIAA Journal, Vol. 47, No. 12, December 2009, pp. 2836-2844.

${ }^{11}$ Eversman, W. and Gallman, J. M., "Impedance Eduction with an Extended Search Procedure," AIAA Paper 2009-3235, May 2009.

${ }^{12}$ Watson, W. R. and Jones, M. G., "New Numerical Procedure for Impedance Eduction in Ducts Containing Mean Flow," AIAA Journal, Vol. 49, No. 10, October 2011, pp. 2109-2122.

${ }^{13}$ Parrott, T. L. and Jones, M. G., "Assessment of NASA's Aircraft Noise Prediction Capability, Chapter 6: Uncertainty in Acoustic Liner Impedance Measurement and Prediction," NASA TP 2012-215653, July 2012.
} 
${ }^{14}$ Watson, W. R. and Nark, D. M., "Assessment of NASA's Aircraft Noise Prediction Capability, Chapter 6: Assessment of Acoustic Propagation and Radiation Codes for Locally Reacting Liners in Flow Ducts," NASA TP 2012-215653, July 2012.

${ }^{15}$ Busse-Gerstengarbe, S., Bake, F., Enghardt, L., and Jones, M. G., "Comparative Study of Impedance Eduction Methods, Part 1: DLR Tests and Methodology," AIAA Paper 2013-2124, May 2013.

${ }^{16}$ Jones, M. G. and Watson, W. R., "Validation of an Improved Experimental Method for Use in Impedance Eduction," AIAA Journal, Vol. 51, No. 1, January 2013, pp. 186-199.

${ }^{17}$ Primus, J., Piot, E., Simon, F., Jones, M., and Watson, W., "ONERA-NASA cooperative effort on liner impedance eduction," AIAA Paper 2013-2273, May 2013.

${ }^{18}$ Hersh, A. S., Jones, M. G., Watson, W. R., and Reilly, S., "Grazing Flow Wedge Resonator Impedance Model," AIAA Paper 2012-2295, June 2012.

${ }^{19}$ Watson, W. R., Carpenter, M. H., and Jones, M. G., "Performance of Kumaresan and Tufts Algorithm in Liner Impedance Eduction with Flow," AIAA Journal, Vol. 53, No. 4, April 2015, pp. 1091-1102.

${ }^{20}$ Myers, M. K., "On the Acoustic Boundary Condition in the Presence of Flow," Journal of Sound and Vibration, Vol. 71, No. 3, 1980, pp. 429-434.

${ }^{21}$ G. W. Stewart, I., "A Modification of Davidon's Minimization Method to Accept Difference Approximations of Derivatives," Journal of ACM, Vol. 14, No. 1, 1967, pp. 72-83.

${ }^{22}$ Coleman, H. W. and Steele, W. G., Experimentation and Uncertainty Analysis for Engineers, John Wiley \& Sons, 1989. 